\title{
Tropospheric response to stratospheric perturbations in a relatively simple general circulation model
}

\author{
Lorenzo M. Polvani ${ }^{1}$ \\ Atmospheric and Oceanic Sciences Program, Princeton University, Princeton, New Jersey, USA
}

Paul J. Kushner

NOAA/Geophysical Fluid Dynamics Laboratory, Princeton, New Jersey, USA

Received 26 October 2001; revised 10 January 2002; accepted 10 January 2002; published 10 April 2002.

[1] The sensitivity of the tropospheric extratropical circulation to thermal perturbations of the polar stratosphere is examined in a dry primitive equation general circulation model with zonally symmetric forcing and boundary conditions. For sufficiently strong cooling of the polar winter stratosphere, the winter-hemisphere tropospheric jet shifts polewards and strengthens markedly at the surface; this is accompanied by a drop in surface pressure at high latitudes in the same hemisphere. In addition, this extratropical tropospheric response is found to be very similar to the model's leading pattern of internal variability. These results are tested for robustness at several horizontal and vertical resolutions, and the same tropospheric response is observed at all but the lowest resolution tested. The behavior of this relatively simple model is broadly consistent with recent observational and modeling studies of trends in extratropical atmospheric variability. INDEX TERMS: 1620 Global Change: Climate Dynamics (3309); 3319 Metrology and Atmospheric Dynamics: General Circulation; 3362 Metrology and Atmospheric Dynamics: Stratosphere/troposphere interactions; 3367 Metrology and Atmospheric Dynamics: Theoretical modeling

\section{Introduction}

[2] Whether and to what degree the stratosphere plays a role in the surface climate and its variability is a question that has lately been receiving much attention (see, e.g., Hartmann et al. [2000] for a recent discussion).

[3] On the observational side, the leading patterns of atmospheric variability - apparently extending from sea level all the way into the stratosphere - are showing amplitude trends that appear to be highly correlated with recent trends in surface temperature in the northern high latitudes [Hurrell, 1995; Thompson and Wallace, 1998]. However, a clear dynamical picture of how the stratosphere might be coupled to the tropospheric circulation is still missing.

[4] On the modeling side, the situation is also unclear. One study has suggested that an adequate resolution of the stratosphere is necessary to simulate the above trends [Shindell et al., 1999], while another study seems to reject this conclusion [Fyfe et al., 1999]. Unfortunately, the complexity of comprehensive general circulation models makes it nearly impossible to independently reproduce the specific results of any one model, or to ascertain the robustness of such results to variations in model parameters.

[5] With reproducibility and robustness as primary concerns, we here employ a relatively simple atmospheric model to answer the

${ }^{1}$ On leave from the Department of Applied Physics and Applied Mathematics and the Department of Earth and Environmental Sciences, Columbia University, New York, New York.

Copyright 2002 by the American Geophysical Union. 0094-8276/02/2001GL014284 following question: If the stratospheric temperature is directly perturbed in a controlled manner, can one detect corresponding changes in the tropospheric circulation? As we will show, our numerical experiments suggest that the answer is yes.

\section{Method}

[6] Our numerical model solves the dry hydrostatic primitive equations in $\sigma$ coordinates, using a spectral-transform method in the horizontal, a Simmons-Burridge finite-difference method in the vertical, and an Asselin-filtered semi-implicit leapfrog scheme for time integration; these techniques are completely standard. The model is forced with a Newtonian relaxation of the temperature field to a prescribed equilibrium profile, which is taken to be a function of latitude and pressure alone. Since the model has no topography, or other longitudinally varying forcings, it is free of stationary planetary waves.

[7] To ensure future reproducibility, all model equations and parameter values are taken to be identical to those in Held and Suarez [1994] (hereafter referred to as HS94), unless explicitly stated. The two main differences between our model and HS94 are the location of the model levels (detailed in the Appendix) and the choice of the equilibrium temperature profile $T_{e q}$, which we take to be of the form

$$
T_{e q}(p, \phi)=\left\{\begin{array}{lll}
T_{e q}^{\text {trop }}(p, \phi) & \text { for } & p \geq p_{T} \\
T_{e q}^{\text {strat }}(p, \phi) & \text { for } & p<p_{T}
\end{array}\right.
$$

where $p$ is the model pressure, $\phi$ the latitude, and $p_{T}=100 \mathrm{mb}$ a nominal tropopause height. The precise analytic forms are given in the Appendix, together with all other numerical details.

[8] In the troposphere, baroclinic eddies are generated via an equator-to-pole temperature gradient maintained by $T_{e q}^{\text {trop }}$, which is chosen to be nearly identical to the one in HS94, the only minor difference being a term that breaks the hemispheric symmetry and strengthens the tropospheric jet in the winter hemisphere.

[9] In the stratosphere, $T_{e q}^{\text {strat }}$ varies smoothly with latitude from a profile with a constant lapse rate $\gamma$ over the winter pole to a profile identical to the U.S. Standard Atmosphere [1976] at lower latitudes and in the summer hemisphere. In our simple model, therefore, the single parameter $\gamma$, whose units are $\mathrm{K} / \mathrm{km}$, allows us to directly control the stratospheric temperature and thus the strength of the polar vortex.

[10] Finally, a sponge layer is used in the top levels of the model to prevent spurious reflection (the explicit form is given in the Appendix). In all figures below, results and analysis are shown for the region below the sponge layer.

\section{Results}

[11] In Figure 1, the zonally averaged zonal winds of five experiments are presented. Each experiment is integrated 10,000 days, with 40 levels in the vertical, at a horizontal resolution of T42 (whose Gaussian grid is roughly $3^{\circ} \times 3^{\circ}$ ), and with a time step of $600 \mathrm{~s}$. An initial spin-up period of 1,000 days is discarded, leaving 

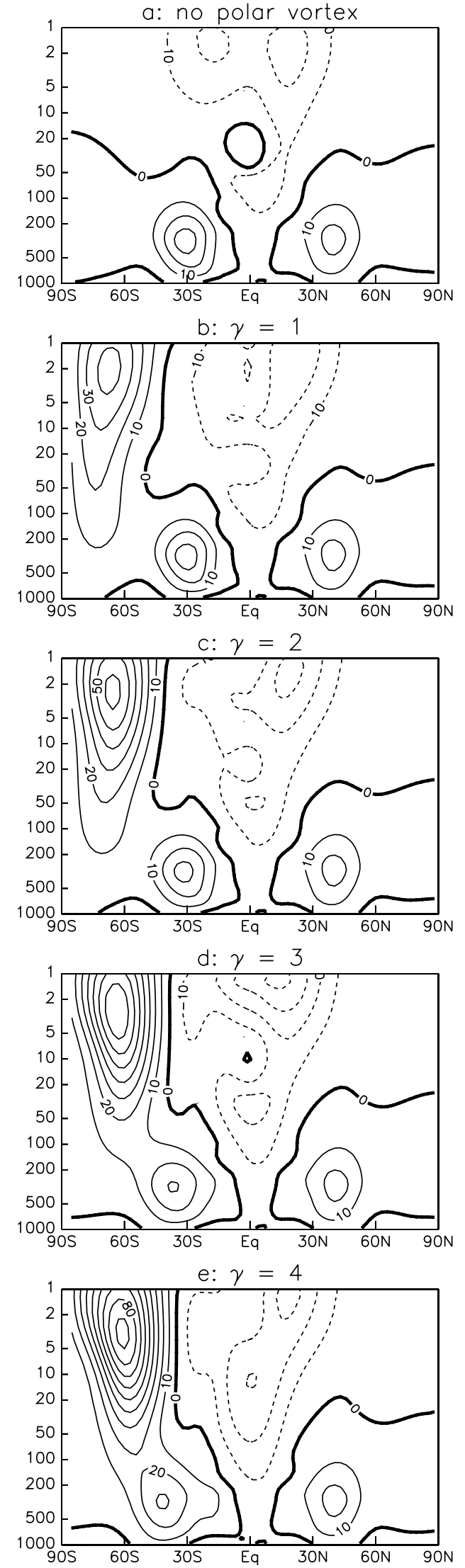

Figure 1. The time and zonally averaged zonal wind, as a function of latitude and pressure, for the experiment with a) no polar vortex in the stratosphere, b) $\gamma=1$, c) $\gamma=2$, d) $\gamma=3$, e) $\gamma=4$. The contour level is $10 \mathrm{~m} \mathrm{~s}^{-1}$. Negative contours are dashed.

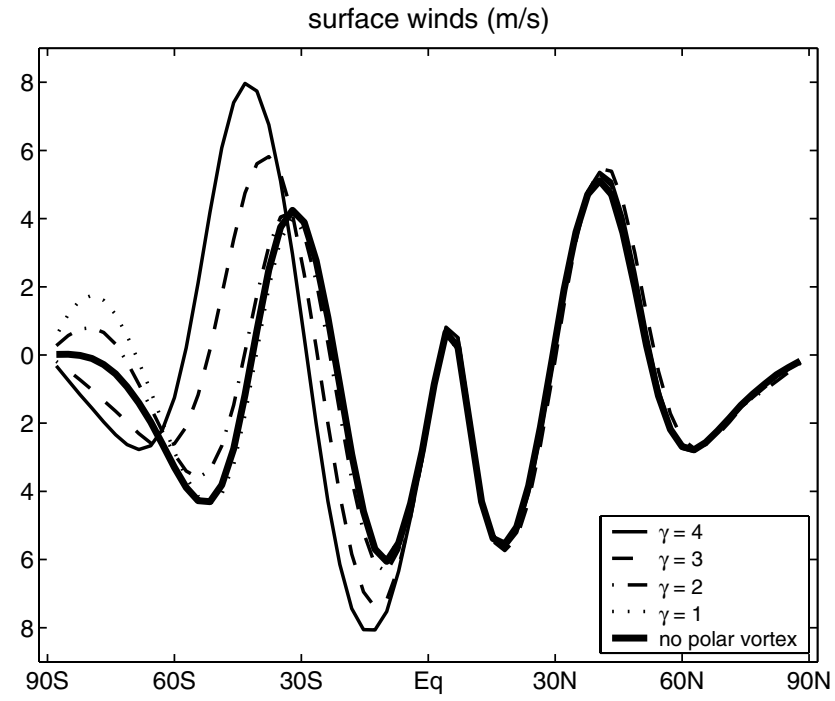

Figure 2. The time and zonally averaged zonal surface wind, in $\mathrm{m} \mathrm{s}^{-1}$, for the five experiments in Figure 1.

almost 25 years of data for each integration. Long integrations are needed to ensure that the model climate is statistically stationary.

[12] In the absence of a polar vortex (Figure 1a), the only distinguishing features of the model climate are the tropospheric jets; the stratosphere is largely quiescent, except for weak easterlies at low latitudes. Predictably, as the polar stratospheric cooling lapse rate $\gamma$ is increased (Figures $1 b-d$ ), the stratospheric jet associated with the polar vortex strengthens, the top stratospheric wind speed growing from $44 \mathrm{~m} \mathrm{~s}^{-1}$ at $\gamma=1$ to $93 \mathrm{~m} \mathrm{~s}^{-1}$ at $\gamma=4$. Animations show that the polar vortex is far from stationary, owing to upwardly propagating transient waves generated by the nonlinear interactions of baroclinic waves in the troposphere, as noted in a study with a similar model [Scinocca and Haynes, 1998]. Given the absence of planetary waves, the strong-vortex cases in Figure 1 may be thought of as a zeroth-order model of the austral winter.

[13] The novel result is that, accompanying the strengthening of the stratospheric polar vortex with increasing $\gamma$, the tropospheric jet shifts polewards in the winter hemisphere: it is centered around $30^{\circ} \mathrm{S}$ at $\gamma=1$ and moves to $45^{\circ} \mathrm{S}$ at $\gamma=4$. Note that the tropospheric jet in the summer hemisphere remains unchanged. We stress that the five numerical experiments in Figure 1 are identical in every respect, except for the thermal relaxation $T_{e q}^{\text {strat }}$ in the stratosphere. Therefore, at least within the context of this relatively simple model, it would seem that thermal perturbations in the polar stratosphere are able to induce clear shifts in the tropospheric circulation.

[14] To bring out this result more clearly, we plot in Figure 2 the time-averaged surface winds for the above five experiments. While a weaker polar vortex seems to have little effect $(\gamma=1,2)$, a stronger polar vortex clearly leads to a stronger westerly surface jet that is shifted polewards $(\gamma=3,4)$, in the winter hemisphere. As $\gamma$ varies from 1 to 4, surface pressure correspondingly drops by 3.1, 4.4, 9.5 and $16.6 \mathrm{mb}$ over the winter polar cap $\left(\phi<60^{\circ} \mathrm{S}\right)$ in the model.

[15] To assess the robustness of the above results, we have performed similar sets of 5 experiments with 20,40 and 80 vertical levels, and with two horizontal resolutions, T21 and T42. The results from these 30 experiments (all integrated for 10,000 days) are summarized in Figure 3. The top panels show the strength of the surface wind increasing in the presence of a stronger polar vortex, and the bottom panels show the surface wind maximum shifting polewards, in all cases except the lowest resolution one, T21 with 20 vertical levels (denoted by circles in panels a and c). This result appears consistent with the conclusion of a previous study [Shindell et al., 1999]: even with a coarse horizontal resolution the effect of stratospheric perturbations can be detected in the troposphere, provided the vertical resolution is sufficiently high. 
a: T21

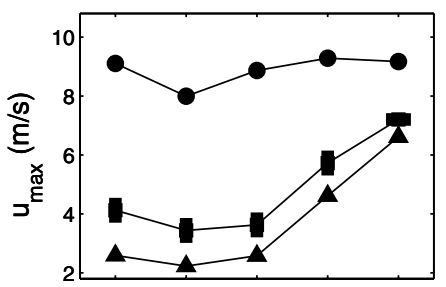

c: T21

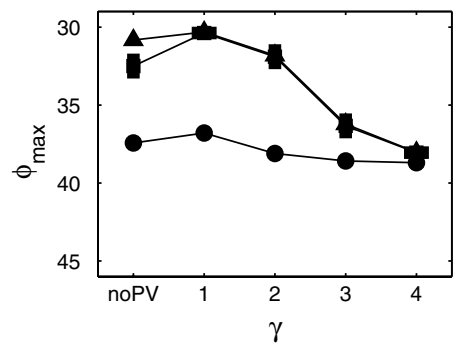

b: T42

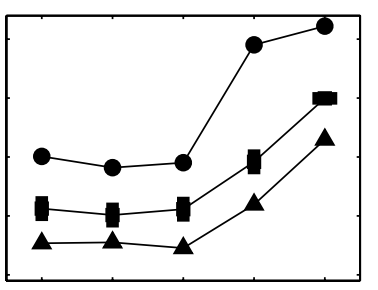

d: T42

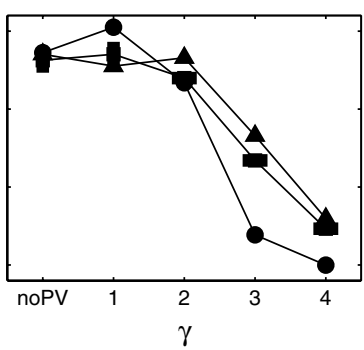

Figure 3. The surface wind maximum $u_{\max }$ in the winter hemisphere, in $\mathrm{m} \mathrm{s}^{-1}$, and its latitudinal location $\phi_{\max }$. Panels a) and c) are for the T21 experiments, panels b) and d) are for the T42 experiments. Circles, squares and triangles indicate experiments with 20, 40, and 80 levels, respectively.

[16] Next, we relate the tropospheric response to the variability of the circulation in our model. Taking the experiment $\gamma=3$ (Figure 1d) as the reference case, we compute the leading EOF of zonally averaged daily sampled surface pressure polewards of $20^{\circ} \mathrm{S}$, weighted by $\sqrt{\cos \phi}$. We find that it accounts for $84 \%$ of the variance (the next EOF accounting for a mere $9.8 \%$ ) and that it captures the familiar seesaw pattern in the winter hemisphere [Thompson and Wallace, 1998]. From the temporal component of this leading EOF we obtain a time series, which we refer to as "the PS1 index," and which is normalized to unit variance.

[17] The zonally averaged zonal winds for the $\gamma=3$ experiment, regressed onto the PS1 index, are shown in Figure 4a. Note the dipolar pattern, with the maxima on either side of the tropospheric jet core, indicating that the main variability of the tropospheric jet consists in a latitudinal wobbling, again in qualitative agreement with observations [Lorenz and Hartman, 2001]. The pattern of the difference in zonal winds between the cases $\gamma=4$ and $\gamma=2$, shown in Figure 4b, is very similar to the wind-regression pattern below $100 \mathrm{mb}$. The similarity between the internal variability and changes in the mean state is consistent with recent modeling and observational studies.

[18] Following Kushner et al. [2001], the "residual" is obtained by removing a scaled version of Figure $4 a$ from Figure $4 b$ (see caption for details). The residual, shown in Figure $4 c$, is largely confined to the stratosphere. This suggests that the response may be separated into a direct, stratospheric component, consisting of a stronger and larger polar vortex, and an indirect, tropospheric component, consisting of a poleward shift of the tropospheric jet.

[19] Our dynamical understanding of this two-component response is, however, far from complete. One might be tempted to invoke a simple "downward-control" mechanism [Haynes et al., 1991], in which changes in the Eliassen-Palm flux divergence in the stratosphere would drive changes in the tropospheric circulation directly below. However, the change in the EliassenPalm flux divergence between the $\gamma=2$ and 4 cases (shown in Figure 5) is modest in the stratosphere and located polewards of the tropospheric jets. This suggests that a more complex mechanism may be at play, involving an initial adjustment of the lower stratosphere, followed by an adjustment of the rest of the troposphere.

[20] In conclusion, we have shown that, within the limitations of a relatively simple general circulation model, externally imposed thermal perturbations in the stratosphere are able to induce a significant tropospheric response. Our simple model's behavior is qualitatively similar to observations, notably in the relation between the tropospheric response and the internal variability of the model [Thompson and Wallace, 1998; Thompson et al., 2000]. Although the dynamical mechanism of this response may be subtle, we hope that it is within reach, given the relative simplicity of our model.

\section{Appendix}

[21] Since we are interested in resolving the stratospheric circulation, a sufficient number of $\sigma$ levels need to be located in the stratosphere. We have used the following scheme: given an integer $n$, let $s_{i}=(i / n)^{5}$, for $0 \leq i \leq n$; the level interfaces of the model are all the values of $s_{i} \geq 10^{-5}$ plus the value $s_{0}=0$; the model levels are then located using the Simmons and Burridge [1981] scheme. $\mathrm{a}: U(\gamma=3)$ regressed on PS1 index

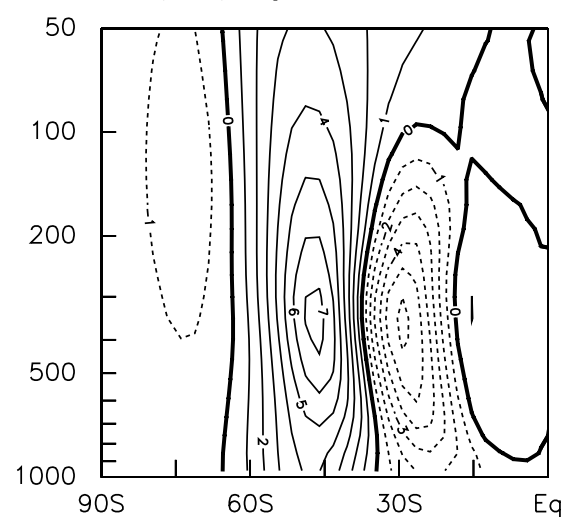

b: $U(\gamma=4)-U(\gamma=2)$

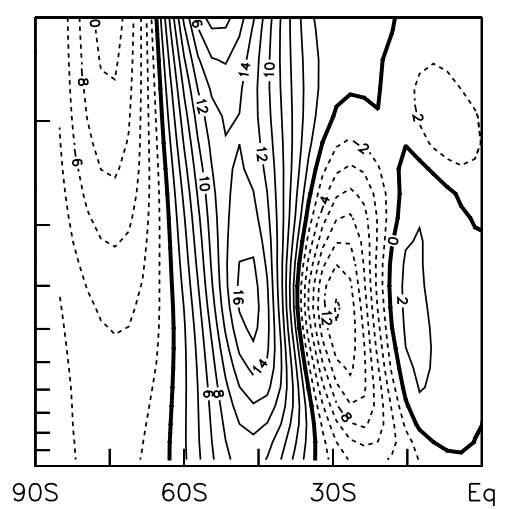

$c: b-2.17 a$

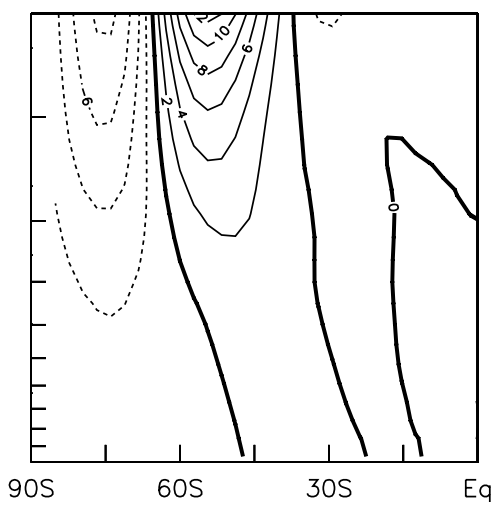

Figure 4. a) The zonal winds regressed onto the PS1 index, in $\mathrm{m} \mathrm{s}^{-1}$, for the experiment of Figure 1d. b) The difference in zonal winds between the experiments in Figure 1e and Figure 1c. c) The residual, defined as the wind-difference (b) minus 2.17 times the regression pattern (a). The factor of 2.17 is obtained by a least-squares fit of the surface wind difference to the surface wind-regression pattern, poleward of $20^{\circ}$. 


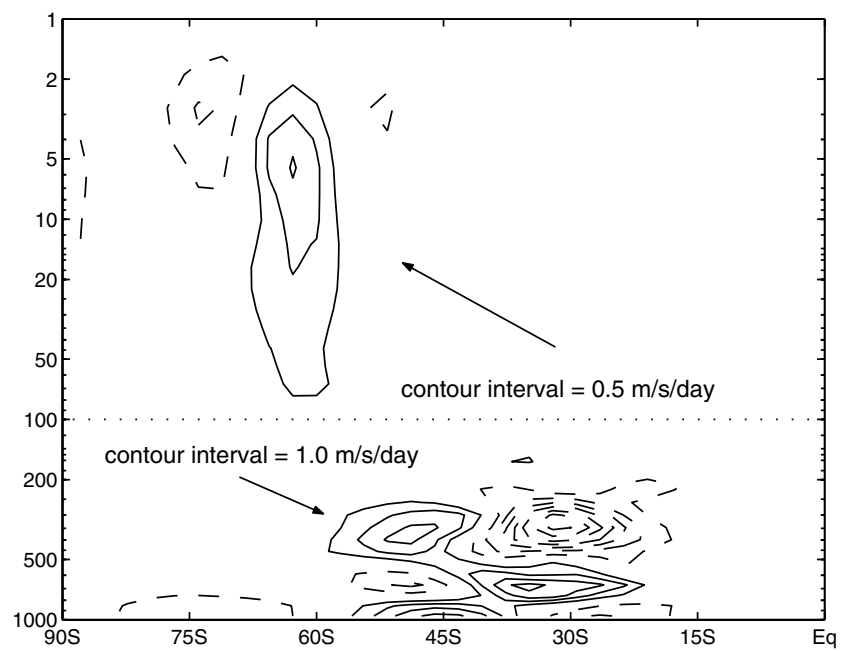

Figure 5. The difference in the zonal-mean eddy-driven zonal acceleration, as a function of latitude and pressure, calculated from the quasi-geostrophic Eliassen-Palm flux divergence, between the $\gamma=4$ and $\gamma=2$ experiments. The contour interval is $1 \mathrm{~m} \mathrm{~s}^{-1}$ day $^{-1}$ below the dotted line, and $0.5 \mathrm{~m} \mathrm{~s}^{-1}$ day $^{-1}$ above it. Negative contours are dashed.

[22] The stratospheric relaxation temperature is given by

$$
T_{e q}^{\text {strat }}(p, \phi)=[1-W(\phi)] T_{U S}(p)+W(\phi) T_{P V}(p)
$$

where $T_{U S}$ is the U.S. Standard Temperature (1976), $T_{P V}(p)=T_{U S}\left(p_{T}\right)\left(p / p_{T}\right)^{-R_{\gamma / g}}$ is the temperature of an atmosphere with a constant lapse rate $\gamma$, and $W(\phi)$ is a weight function used to confine the cooling over the winter pole,

$$
W(\phi)=(1 / 2)\left(1-\tanh \left[\left(\phi-\phi_{0}\right) / \delta \phi\right]\right)
$$

with $\phi_{0}=-50$ and $\delta \phi=10$. The no-polar-vortex case is obtained by setting $W(\phi)=0$.

[23] The tropospheric relaxation temperature is given by

$$
T_{e q}^{\text {trop }}(p, \phi)=\max \left[T_{T},\left(T_{0}-\delta T\right)\left(p / p_{0}\right)^{\kappa}\right],
$$

where $T_{0}=315 \mathrm{~K}, p_{0}=1000 \mathrm{mb}$, and $\kappa=2 / 7$, with

$$
\delta T=\delta_{y} \sin ^{2} \phi+\epsilon \sin \phi+\delta_{z} \log \left(p / p_{0}\right) \cos ^{2} \phi
$$

where $\delta_{y}=60 \mathrm{~K}, \delta_{z}=10 \mathrm{~K}$, and $\epsilon=10 \mathrm{~K}$. The nonzero value of $\epsilon$ provides a simple asymmetry between the winter and summer hemispheres. Continuity of $T_{e q}$ at $p=p_{T}$ results from the choice $T_{T}=T_{U S}\left(p_{T}\right)$.
[24] The sponge layer is applied as a linear damping term on the momentum equations, with a damping coefficient $k_{s p}=0$ for $p \geq p_{s p}$, and $k_{s p}(p)=k_{\max }\left[\left(p_{s p}-p\right) / p_{s p}\right]^{2}$ for $p<p_{s p}$. We have used $p_{s p}=0.5 \mathrm{mb}$ and $k_{\max }=0.5$ day $^{-1}$.

[25] In order to minimize numerical diffusion, we have used a $\nabla^{6}$ hyperviscosity, with a diffusion time scale of half a day on the largest resolved wavenumber.

[26] For future reference, we note that the code used for this study is based on a pre-release ("damascus") version of the NOAA/GFDL Flexible Modeling System spectral dynamical core.

[27] Acknowledgments. We thank John Wilson, John Fyfe, and Ted Shepherd for useful comments on the manuscript, and Isaac Held for insightful discussions. LMP is funded by the National Science Foundation.

\section{References}

Fyfe, J. C., G. J. Boer, and G. M. Flato, The Arctic and Antarctic Oscillations and their projected changes under global warming, Geophys. Res. Lett., 26, 1601-1604, 1999.

Hartmann, D. L., J. M. Wallace, V. Limpasuvan, D. W. J. Thompson, and J. R. Holton, Can ozone depletion and global warming interact to produce rapid climate change?, Proc. Nat. Acad. Sci. USA, 97, 1412-1417, 2000.

Haynes, P. H., C. J. Marks, M. E. McIntyre, T. G. Shepherd, and K. P. Shine, On the "downward control" of extratropical diabatic circulations by eddy-induced mean zonal forces, J. Atmos. Sci, 48, 651-679, 1991.

Held, I. M., and M. J. Suarez, A proposal for the intercomparison of the dynamical cores of atmospheric general-circulation models, Bull. Am. Meteorol. Soc., 75, 1825-1830, 1994.

Hurrell, J. W., Decadal trends in the North Atlantic Oscillation: regional temperatures and precipitation, Science, 269, 676-679, 1995.

Kushner, P. J., I. M. Held, and T. L. Delworth, Southern Hemisphere atmospheric circulation response to global warming, J. Climate, 14, 22382249, 2001.

Lorenz, D. J., and D. L. Hartman, Eddy-zonal flow feedback in the Southern Hemisphere, J. Atmos. Sci., 58, 3312-3337, 2001.

Scinocca, J. F., and P. H. Haynes, Dynamical forcing of stratospheric planetary wave by tropospheric baroclinic eddies, J. Atmos. Sci., 55, 23612392, 1998.

Shindell, D. T., R. L. Miller, G. A. Schmidt, and L. Pandolfo, Simulation of recent northern winter climate trends by greenhouse-gas forcing, Nature, $399,452-455,1999$

Simmons, A. J., and D. M. Burridge, An energy and angular-momentum conserving vertical finite-difference scheme and hybrid vertical-coordinates, Mon. Wea. Rev., 109, 758-766, 1981.

Thompson, D. W. J., and J. M. Wallace, The Arctic Oscillation signature in the wintertime geopotential height and temperature fields, Geophys. Res. Lett., 25, 1297-1300, 1998.

Thompson, D. W. J., J. M. Wallace, and G. C. Hegerl, Annular modes in the extratropical circulation, Part II: Trends, J. Climate, 13, 1018-1036, 2000.

U.S. Standard Atmosphere, U.S. Government Printing Office, Washington, D.C., 1976.

L. M. Polvani, Columbia University, S.W. Mudd Room 216, 500 West 120th Street, New York, NY 10027, USA. (polvani@columbia.edu)

P. J. Kushner, Geophysical Fluid Dynamics Laboratory, PO Box 308, Forrestal Campus, Princeton, NJ 08542, USA. (pjk@gfdl.noaa.gov) 